\title{
Anti-MOG antibody-positive ADEM following infectious mononucleosis due to a primary EBV infection: a case report
}

Yoshitsugu Nakamura ${ }^{{ }^{* \dagger}}$, Hideto Nakajima ${ }^{1 \dagger}$, Hiroki Tani ${ }^{1}$, Takafumi Hosokawa ${ }^{1}$, Shimon Ishida ${ }^{1}$, Fumiharu Kimura ${ }^{1}$, Kimihiko Kaneko², Toshiyuki Takahashi ${ }^{3}$ and Ichiro Nakashima²

\begin{abstract}
Background: Anti-Myelin oligodendrocyte glycoprotein (MOG) antibodies are detected in various demyelinating diseases, such as pediatric acute disseminated encephalomyelitis (ADEM), recurrent optic neuritis, and aquaporin-4 antibody-seronegative neuromyelitis optica spectrum disorder. We present a patient who developed anti-MOG antibody-positive ADEM following infectious mononucleosis (IM) due to Epstein-Barr virus (EBV) infection.

Case presentation: A 36-year-old healthy man developed paresthesia of bilateral lower extremities and urinary retention 8 days after the onset of IM due to primary EBV infection. The MRI revealed the lesions in the cervical spinal cord, the conus medullaris, and the internal capsule. An examination of the cerebrospinal fluid revealed pleocytosis. Cell-based immunoassays revealed positivity for anti-MOG antibody with a titer of 1:1024 and negativity for anti-aquaporin-4 antibody. His symptoms quickly improved after steroid pulse therapy followed by oral betamethasone. Anti-MOG antibody titer at the 6-month follow-up was negative.

Conclusions: This case suggests that primary EBV infection would trigger anti-MOG antibody-positive ADEM. Adult ADEM patients can be positive for anti-MOG antibody, the titers of which correlate well with the neurological symptoms.
\end{abstract}

Keywords: Myelin oligodendrocyte glycoprotein, Acute disseminate encephalomyelitis, Epstein-Barr virus, Transverse myelitis, Antecedent infection, Case report

\section{Background}

Myelin-oligodendrocyte glycoprotein (MOG) is exclusively expressed on the surface of oligodendrocytes in the central nervous system (CNS). Anti-MOG antibody is predominantly detected in pediatric acute disseminated encephalomyelitis (ADEM), recurrent optic neuritis, and aquaporin-4 antibody-seronegative neuromyelitis optica spectrum disorder (NMOSD). Recent studies proposed that anti-MOG antibodyassociated demyelinating diseases were indeed a clinical spectrum in pediatric patients and that their clinical features were different from those of multiple sclerosis and NMOSD with anti-aquaporin-4 (AQP4)

\footnotetext{
* Correspondence: in1394@osaka-med.ac.jp

${ }^{\dagger}$ Equal contributors

${ }^{1}$ Division of Neurology, Department of Internal Medicine IV, Osaka Medical

College, Daigakumachi 2-7, Takatsukishi, Osaka 569-8686, Japan

Full list of author information is available at the end of the article
}

antibody [1, 2]. ADEM is a heterogeneous syndrome that is occasionally triggered by an antecedent infection [3]. A patient with anti-MOG antibody-positive longitudinally extensive transverse myelitis (LTEM) that developed after infection with influenza virus was previously reported [4]. However, no anti-MOG antibody-positive ADEM cases with a preceding viral infection other than influenza have been reported till date. Here we present a patient who developed antiMOG antibody-positive ADEM following infectious mononucleosis (IM) due to primary Epstein-Barr virus (EBV) infection.

\section{Case presentation}

A 36-year-old healthy man developed fever and right cervical lymphadenopathy. Laboratory analysis showed elevated white blood count $\left(10,390 / \mathrm{mm}^{3}\right.$ with $33 \%$ 
neutrophil, 51\% lymphocyte, and 12\% atypical lymphocytes), elevated liver enzymes (aspartate transaminase, $193 \mathrm{U} / \mathrm{l}$; alanine transaminase, $413 \mathrm{U} / \mathrm{l})$. Serological studies indicated primary EBV infection (EBV viral capsid antigen [VCA] IgM, positive at 1:40; EBV VCA IgG, positive at 1:160, EBV nuclear antigen IgG, negative). Serologic testing for human immunodeficiency virus antibody was negative. Based on these clinical features, the patient was diagnosed with IM due to primary EBV infection. However, 8 days after onset, the patient developed paresthesia of bilateral lower extremities and urinary retention, which were exacerbated over the next few days. The patient was alert and oriented but had a high fever of $38.5{ }^{\circ} \mathrm{C}$. Neurological examination revealed normal cranial nerves and no weakness in limbs; however, unstable gait with hyperreflexia, sensory disturbance in the entire area below the T7 level, and dysuria that required urethral catheterization were present. Laboratory analysis showed normal white blood count and decreasing liver enzyme levels. Antinuclear and SS-A antibody levels were within normal limits. Cerebrospinal fluid (CSF) examination showed pleocytosis (76/ $\mathrm{mm}^{3}$ ), protein concentration of $104.3 \mathrm{mg} / \mathrm{dl}$, IgG index of 0.61 , the absence of oligoclonal IgG bands. In addition, IgG and IgM antibodies to EBV VCA and polymerase chain reaction for EBV DNA were negative in the CSF. These findings excluded the direct presence of EBV in the CNS. Additionally, polymerase chain reaction for herpes simplex virus 1, herpes simplex virus 2, and varicellazoster virus DNA were negative in the CSF. IgG and IgM antibodies to cytomegalovirus were negative in the CSF. These findings excluded viral myelitis. Spinal MRI showed a T2-hyperintense lesion predominantly in the central gray matter extending from C2 to C6 (Fig. 1). Brain MRI showed a fluid-attenuated inversion recovery-hyperintense lesion in the left posterior limb of the internal capsule (Fig. 1). Nerve conduction studies of the left upper and lower extremities showed normal motor and sensory function. Cell-based immunoassays revealed positivity for anti-MOG antibody with a titer of 1:1024 and negativity for anti-AQP4 antibody [2]. Therefore, the patient was started on immunosuppressive therapy with intravenous methylprednisolone (IVMP) for 3 consecutive days, followed by oral betamethasone ( $2 \mathrm{mg} /$ day). The gadolinium-enhanced spinal MRI after the start of therapy revealed slight gadolinium enhancement of the conus medullaris surface (Fig. 1). However, shortly after IVMP initiation, his symptoms demonstrated significant improvement, and urethral catheter was removed 9 days after the start of IVMP. His sensory disturbance and gait instability was completely resolved 2 weeks after IVMP initiation. Oral betamethasone was tapered following IVMP, and he was discharged without any symptoms or sequelae. Follow-up MRI 1 month after IVMP showed reduction in all CNS lesions. Anti-MOG antibody titer at the 6-month follow-up was negative.

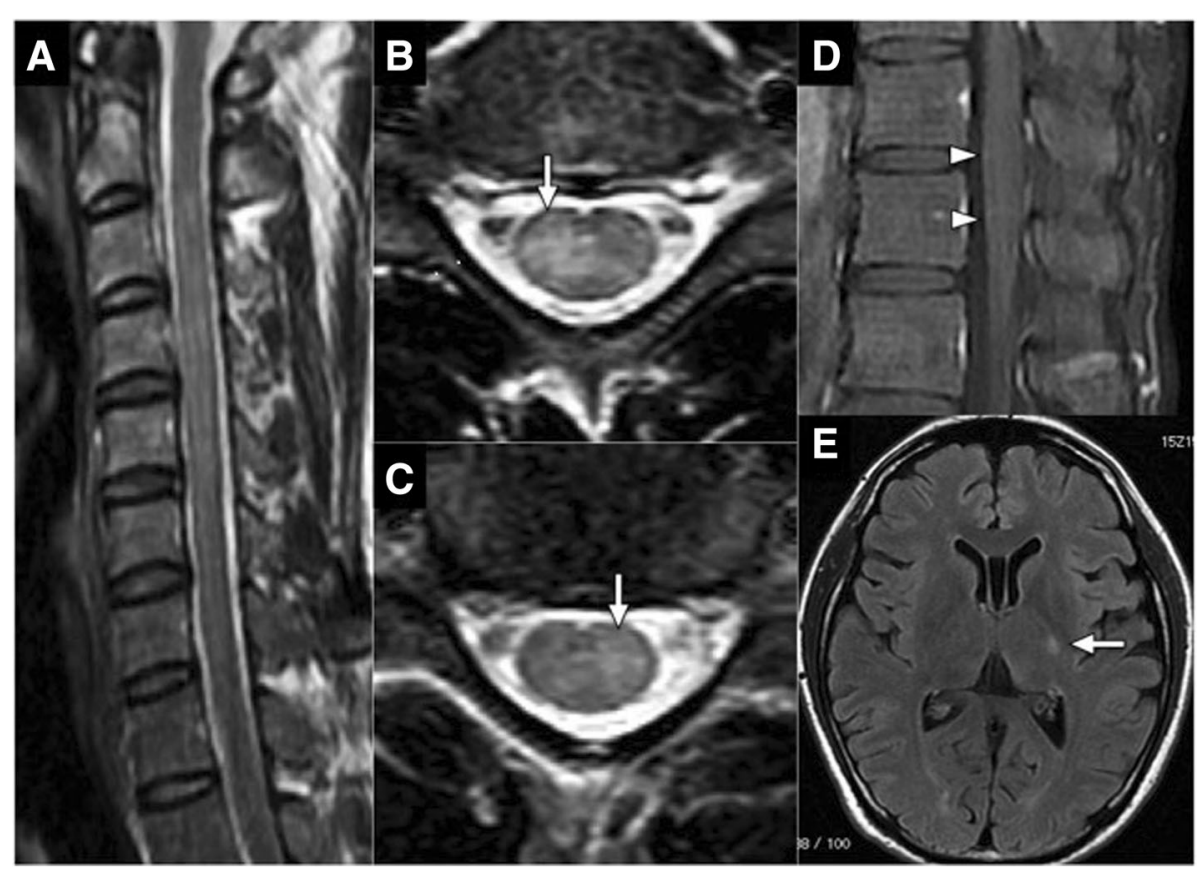

Fig. 1 Spinal cord T2-weighted MRI shows a hyperintense lesion extending from C2 to C6 on the sagittal view (a), and predominantly in the central gray matter on the axial view at C4 (b. arrow) and C6 level (c. arrow). Gadolinium-enhanced T1-weighted MRI shows slight gadolinium enhancement within the conus medullaris surface (d. arrow heads). Brain fluid-attenuated inversion recovery MRI shows a hyperintense lesion in the left crus posterius capsulae internae (e. arrow) 
No symptomatic recurrence was observed during followup evaluation at 11 months after onset. Clinical course, the CSF and MRI findings, and the response to immunosuppressive therapy were most consistent with the diagnosis of anti-MOG antibody-positive ADEM $[3,5]$.

\section{Discussion and Conclusions}

We present a case of a patient who developed antiMOG antibody-positive ADEM following IM. In our patient, ADEM occurred relatively early i.e., 8 days after IM onset. However, the absence of EBV genome in the CSF samples is strong evidence for an autoimmune pathogenesis of neurological signs following IM. The present case illustrates two important clinical issues. First, adult ADEM patients can be positive for antiMOG antibody, the titers of which correlated well with neurological symptoms. Among pediatric ADEM cases, pleocytosis, spinal cord lesions characterized by LTEM, and better outcomes were observed more frequently in patients with anti-MOG antibody than in those with negative titers. Anti-MOG antibody titers of monophasic ADEM patients declined or became negative over the course of months to years [6]. However, patients with persistently high anti-MOG antibody titers experienced a recurrent disease course [6, 7]. The anti-MOG antibody titer of the present case became negative and did not show recurrence. Thus, assessment for antiMOG antibody titers in adult ADEM patients might be useful in predicting prognosis and determining the course of disease management.

Second, primary EBV infection triggers anti-MOG antibody-positive ADEM. Antecedent infections were reported in $46 \%$ of adult ADEM patients [3]. However, those were usually nonspecific upper respiratory tract infections, and systemic viral infections preceding ADEM were rarely reported in adult patients [3]. While several studies previously reported ADEM and LTEM cases associated with EBV infection [8-14], anti-MOG antibody titers were not examined in any of the studies. Recent reports proposed the presence of cross-reactivity between EBV and myelin proteins [15] and between MOG and EBV nuclear antigen [16]. Anti-MOG antibody was detected in $20 \%$ of patients with IM due to primary EBV infection without neurological manifestations [17]. These findings implicate EBV infection as a potential trigger for anti-MOG antibody production. However, a potential specific molecular mimicry between antibodies produced in response to EBV antigens and MOG remains unclear. The incidence of neurological involvement in IM was reported to range be $0.37-7.3 \%$ [8], and LTEM and ADEM remain very rare complications of EBV infection [8-10]. Therefore, we propose that anti-MOG antibody production might result from synergistic effects of additional unknown factors in response to EBV infection.
In conclusion, this case highlights the possibility that primary EBV infection triggers anti-MOG antibodypositive ADEM. Future studies are necessary to confirm the role of EBV in the pathogenesis of anti-MOG antibody-associated demyelinating diseases.

\section{Abbreviations \\ ADEM: Acute disseminated encephalomyelitis; AQP4: Aquaporin-4; \\ CNS: Central nervous system; CSF: Cerebrospinal fluid; DNA: Deoxyribonucleic acid; EBV: Epstein-Barr virus; IM: Infectious mononucleosis; IVMP: Intravenous methylprednisolone; LTEM: Longitudinally extensive transverse myelitis; MOG: Myelin oligodendrocyte glycoprotein; MRI: Magnetic resonance imaging; NMOSD: Neuromyelitis optica spectrum disorder; VCA: Viral capsid antigen}

\section{Acknowledgments}

Not applicable.

\section{Funding}

This work was supported by JSPS KAKENHI, Grant Number 15 K45678, from the Ministry of Education, Culture, Sports, Science and Technology, Japan. The funders had no role in the study design, data collection and analysis, decision to publish, or preparation of the manuscript.

Availability of data and materials

The dataset supporting the conclusion of this article is included within the article.

Authors' contributions

YN and HN examined and scripted the manuscript. HT, TH, SI, and FK helped to draft the manuscript and performed analyses. KK and $\Pi$ performed anti-MOG antibody analysis. IN supported for the critical revision of the manuscript for intellectual content. All authors approved the contents of this case report.

\section{Competing interest}

The authors declare that they have no competing interests.

\section{Consent for publication}

Written informed consent was obtained from the patient for publication of this case report and any accompanying images.

Ethics approval and consent to participate

The authors declare that ethics approval was not required for this case report.

\section{Publisher's Note}

Springer Nature remains neutral with regard to jurisdictional claims in published maps and institutional affiliations.

\section{Author details}

${ }^{1}$ Division of Neurology, Department of Internal Medicine IV, Osaka Medical College, Daigakumachi 2-7, Takatsukishi, Osaka 569-8686, Japan. ²Division of Neurology, Tohoku University School of Medicine, Seiryomachi 1-1, Aobaku, Sendai 980-8574, Japan. ${ }^{3}$ Division of Neurology, NHO Yonezawa Hospital, Ozimisawa 26100-1, Yonezawashi, Yamagata 992-1202, Japan.

Received: 21 December 2016 Accepted: 11 April 2017

Published online: 19 April 2017

References

1. Reindl M, Di Pauli F, Rostásy K, Berger T. The spectrum of MOG autoantibody-associated demyelinating diseases. Nat Rev Neurol. 2013;9: 455-61.

2. Sato DK, Callegaro D, Lana-Peixoto MA, Waters PJ, de Haidar Jorge FM, Takahashi T, et al. Distinction between MOG antibody-positive and AQP4 antibody-positive NMO spectrum disorders. Neurology. 2014;82:474-81.

3. Schwarz S, Mohr A, Knauth M, Wildemann B, Storch-Hagenlocher B. Acute disseminated encephalomyelitis: a follow-up study of 40 adult patients. Neurology. 2001;56:1313-8. 
4. Amano H, Miyamoto N, Shimura H, Sato DK, Fujihara K, Ueno S, et al. Influenza-associated MOG antibody-positive longitudinally extensive transverse myelitis: a case report. BMC Neurol. 2014;14:224.

5. Tenembaum S, Chitnis T, Ness J, Hahn JS, International Pediatric MS Study Group. Acute disseminated encephalomyelitis. Neurology. 2007;68(16 Suppl 2):S23-36.

6. Baumann M, Sahin K, Lechner C, Hennes EM, Schanda K, Mader S, et al. Clinical and neuroradiological differences of paediatric acute disseminating

encephalomyelitis with and without antibodies to the myelin oligodendrocyte glycoprotein. J Neurol Neurosurg Psychiatry. 2015;86:265-72.

7. Baumann M, Hennes EM, Schanda K, Karenfort M, Kornek B, Seidl R, et al. Children with multiphasic disseminated encephalomyelitis and antibodies to the myelin oligodendrocyte glycoprotein (MOG): extending the spectrum of MOG antibody positive diseases. Mult Scler. 2016;22:1821-9.

8. Silverstein A, Steinberg G, Nathanson M. Nervous system involvement in infectious mononucleosis. The heralding and-or major manifestation. Arch Neurol. 1972;26:353-8.

9. Fujimoto H, Asaoka K, Imaizumi T, Ayabe M, Shoji H, Kaji M. Epstein-Barr virus infections of the central nervous system. Intern Med. 2003:42:33-40.

10. Tselis AC. Epstein-Barr virus infections of the nervous system. Handb Clin Neurol. 2014;123:285-305.

11. Mohsen $\mathrm{H}$, Abu Zeinah GF, Elsotouhy AH, Mohamed K. Acute disseminated encephalomyelitis following infectious mononucleosis in a toddler. BMJ Case Rep 2013; doi:10.1136/bcr-2013-010048.

12. Caldas C, Bernicker E, Nogare AD, Luby JP. Case report: transverse myelitis associated with Epstein-Barr virus infection. Am J Med Sci. 1994;307:45-8.

13. Junker AK, Roland EH, Hahn G. Transverse myelitis and Epstein-Barr virus infection with delayed antibody responses. Neurology. 1991;41:1523-4.

14. Bahadori HR, Williams VC, Turner RP, Rumboldt Z, Reigart JR, Fowler SL, et al. Acute disseminated encephalomyelitis following infectious mononucleosis. J Child Neurol. 2007;22:324-8.

15. Lang HL, Jacobsen H, Ikemizu S, Andersson C, Harlos K, Madsen L, et al. A functional and structural basis for TCR cross-reactivity in multiple sclerosis. Nat Immunol. 2002;3:940-3.

16. Wang $\mathrm{H}$, Munger $\mathrm{KL}$, Reindl $M, \mathrm{O}$ 'Reilly EJ, Levin $\mathrm{LI}$, Berger $\mathrm{T}$, et al. Myelin oligodendrocyte glycoprotein antibodies and multiple sclerosis in healthy young adults. Neurology. 2008;71:1142-6.

17. Kakalacheva K, Regenass S, Wiesmayr S, Azzi T, Berger C, Dale RC, et al. Infectious mononucleosis triggers generation of lgG auto-antibodies against native myelin oligodendrocyte glycoprotein. Viruses. 2016;8:51.

\section{Submit your next manuscript to BioMed Central and we will help you at every step:}

- We accept pre-submission inquiries

- Our selector tool helps you to find the most relevant journal

- We provide round the clock customer support

- Convenient online submission

- Thorough peer review

- Inclusion in PubMed and all major indexing services

- Maximum visibility for your research

Submit your manuscript at www.biomedcentral.com/submit 